\title{
Bariatric surgery reduces CD36-bearing microvesicles of endothelial and monocyte origin
}

\author{
Jaco Botha ${ }^{1,2^{*}}$ (D, Morten Hjuler Nielsen ${ }^{1}$, Maja Høegh Christensen ${ }^{1}$, Henrik Vestergaard ${ }^{3}$ and Aase Handberg ${ }^{1,2}$
}

\begin{abstract}
Background: Bariatric surgery is a widely adopted treatment for obesity and its secondary complications. In the past decade, microvesicles (MVs) and CD36 have increasingly been considered as possible biomarkers for obesity, the metabolic syndrome (MetSy), type 2 diabetes mellitus (T2DM). Thus, the purpose of this study was to investigate how weight loss resulting from bariatric surgery affects levels of specific MV phenotypes and their expression of CD36 scavenger receptor. Additionally, we hypothesised that subjects with MetSy had higher baseline concentrations of investigated MV phenotypes.

Methods: Twenty individuals undergoing Roux-en-Y gastric bypass surgery were evaluated before and 3 months after surgery. MVs were characterised by flow cytometry at both time points and defined as lactadherin-binding particles within a 100-1000 nm size gate. MVs of monocyte (CD14) and endothelial (CD62E) origin were defined by cell-specific markers, and their expression of CD36 was investigated.

Results: Following bariatric surgery, subjects incurred an average BMl reduction (delta) of $-8.4 \pm 1.4(p<0.0001)$. Significant reductions were observed for the total MVs $(-66.55 \%, p=0.0017)$ and MVs of monocyte $(-36.11 \%$, $p=0.0056)$ and endothelial $(-40.10 \%, p=0.0007)$ origins. Although the bulk of CD36-bearing MVs were unaltered, significant reductions were observed for CD36-bearing MVs of monocyte $(-60.04 \%, p=0.0192)$ and endothelial $(-54.93 \%, p=0.04)$ origin. No differences in levels of MVs were identified between subjects who presented with MetSy at baseline $(n=13)$ and those that did not $(n=7)$.

Conclusion: Bariatric surgery resulted in significantly altered levels of CD36-bearing MVs of monocyte and endothelial origin. This likely reflects improvements in ectopic fat distribution, plasma lipid profile, low-grade inflammation, and oxidative stress following weight loss. Conversely, however, the presence of MetSy at baseline had no impact on MV phenotypes.
\end{abstract}

Keywords: Extracellular vesicles, CD36, Obesity, Ectopic fat deposition, Metabolic syndrome, Bariatric surgery

\section{Background}

Decades of research indicate that lifestyle interventions and pharmacotherapy of obesity often fail to result in sufficient and sustained reductions in weight to reduce an individual's risk of obesity-related morbidity and mortality [1, 2]. However, a large body of evidence suggests that bariatric surgery can result in sustained

\footnotetext{
* Correspondence: j.botha@rn.dk

${ }^{1}$ Department of Clinical Biochemistry, Aalborg University Hospital, Hobrovej 18-22, DK-9000 Aalborg, Denmark

${ }^{2}$ Department of Clinical Medicine, Faculty of Medicine, Aalborg University, Sdr. Skovvej 15, DK-9000 Aalborg, Denmark

Full list of author information is available at the end of the article
}

weight loss, reduce an individual's risk of type 2 diabetes mellitus (T2DM) recurrence, and decrease levels of circulating inflammatory markers associated with obesity, the metabolic syndrome, and T2DM [3-5]. Therefore, bariatric surgery is adopted to an increasing extent globally as a treatment for morbid obesity and its secondary complications [6-8].

In recent years, a growing number of studies have begun to realise the potential of microvesicles (MVs) as biomarkers for a number of diseases, including the metabolic syndrome (MetSy) [9], T2DM [10], and atherosclerosis [11]. MVs are a subset of extracellular

(c) The Author(s). 2018 Open Access This article is distributed under the terms of the Creative Commons Attribution 4.0 International License (http://creativecommons.org/licenses/by/4.0/), which permits unrestricted use, distribution, and 
vesicles (EVs) that can be distinguished by their biogenesis, namely outwards budding of the plasma membrane and subsequent occlusion and shedding of the particle [12]. Like all other extracellular vesicles, MVs are a heterogeneous group of plasma membrane-enclosed particles containing components from their cellular origin, which are shed by most cell types in latent, activated and apoptotic states [13]. A soluble form of CD36 (sCD36) has previously been demonstrated to be present in circulation, and increased levels have been associated with abdominal fat distribution [14], insulin resistance [15], and non-alcoholic fatty liver disease (NAFLD) [16]. Although the exact nature of sCD36 is unknown, studies have previously revealed that, at least in part, sCD36 is associated with circulating MVs $[11,17]$.

CD36 is a scavenger receptor that has been associated with cellular uptake of lipids in a whole range of cells, and its expression is increased in obesity, MetSy, and diabetes [18-23]. In addition, a large body of evidence suggests that CD36 is involved in pro-inflammatory polarisation of macrophages upon exposure to oxidised LDL cholesterol and therefore contributes to the low-grade inflammatory state observed in diet-induced obesity and MetSy [22, 24]. Thus, measuring CD36 on the surface of MVs might not only yield important information about ectopic fat deposition but also the level of low-grade inflammation.

Therefore, it was hypothesised that significant weight loss and improvement in cardio-metabolic risk factors resulting from bariatric surgery would result in decreased concentrations of circulating MVs, MVs positive for the expression of CD36 on their surface, MVs of monocyte (MMVs) or endothelial (EMV) origin, and MMVs and EMVs positive for CD36. It was further hypothesised that subjects suffering from MetSy at inclusion had increased baseline concentrations of all of the abovementioned MV phenotypes.

\section{Methods}

\section{Study design}

The study population and design have been described elsewhere [14]. In brief, twenty individuals undergoing bariatric surgery were recruited for the present study. All participants met the Danish requirements for referral to bariatric surgery: $\geq 20$ years of age and a body mass index $(\mathrm{BMI}) \geq 40 \mathrm{~kg} \mathrm{~m}-2$ or $\mathrm{BMI} \geq 35$ with associated co-morbidities. Participants were additionally required to lose approximately $8 \%$ of their body weight prior to surgery and inclusion in this study. Bariatric surgery was performed by either one of two surgeons with expertise in Roux-en- $Y$ gastric bypass using a standard laparoscopic Roux-en-Y technique at the Aleris-Hamlet Hospital, Copenhagen, Denmark [14]. All participants were dismissed within $24 \mathrm{~h}$ after surgery and no subjects suffered from post-surgical complications.

All twenty subjects were evaluated prior to (baseline) and three months after bariatric surgery. At each visit, following an overnight fast, height and weight were recorded, fat mass and distribution was measured by full body dual-energy $\mathrm{x}$-ray absorptiometry, and venous blood samples were collected for biochemical analyses and flow cytometric measurement of MVs. Haemoglobin, leukocytes, aspartate aminotransferase (AST), alanine aminotransferase (ALT), glycosylated haemoglobin, plasma glucose, C-peptide, serum insulin, total cholesterol, high-density lipoprotein, low-density lipoprotein, and triglycerides were determined with standardised methods in a routine biochemical laboratory. Plasma soluble CD36 (sCD36) was measured with an in-house ELISA as previously described [15]. Serum YKL40 was determined with a commercial ELISA (Quidel, San Diego, CA, USA). High sensitivity C-reactive protein (hsCRP) was measured with a commercial highly sensitive, latex particle-enhanced immunoturbidimetric assay (DAKO, Glostrup, Denmark). Furthermore, insulin sensitivity was determined with the homeostasis model assessment (HOMA-\%S, http://www.dtu.ox.ac.uk/homacalculator/index.php), and liver fat percentage (LF\%) was predicted using an algorithm based on the presence of MetSy and T2DM as well as fasting insulin and ALT and AST [25].

\section{Flow cytometric analysis of MVs}

Blood samples for flow cytometric analysis of MVs were collected into EDTA tubes and the first centrifugation cycle was initiated within two hours after collection. Samples were subjected to centrifugation at $2000 \mathrm{~g}$ for $10 \mathrm{~min}$ to yield blood plasma. Plasma was stored at $80{ }^{\circ} \mathrm{C}$ until analysis. Flow cytometric analysis of plasma MV content was performed as described previously [26] and in supplementary materials on a BD FACSAria ${ }^{\text {tw }}$ III High Speed Cell Sorter (BD Biosciences, San Jose, CA, USA) and data was analysed in FlowJo $^{\circ}$ version 10.4 (FlowJo LLC, Oregon, USA) as depicted in Fig. 1.

\section{Statistical data analysis}

All statistical data analyses and plotting was performed in and R 3.2.5 (R Core Team, Vienna, Austria) with $x l s x$ [27], ggplot2 [28], and reshape2 [29] packages installed. The assumption of normality was tested using Shapiro-Wilk's W-test and confirmed visually for each parameter using QQ-plots and histograms. Paired Student's t-test or Wilcoxon signed rank test were used to compare pre and post-surgical values for all parameters where appropriate, whereas unpaired Student's t-test or Mann-Whitney $U$ test were utilized to compare baseline parameters with regards to the presence of 


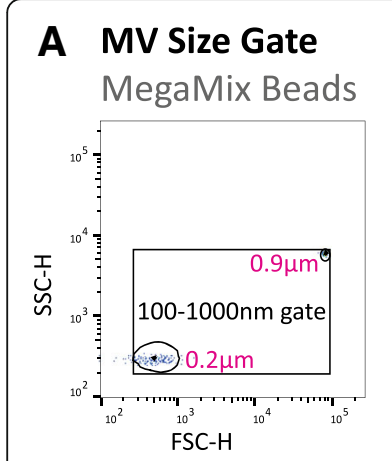

\section{TruCount Beads} Labelled PFP

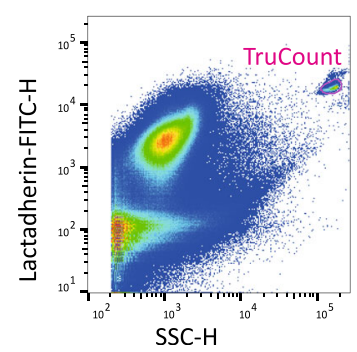

B PS Expression Unlabelled PFP

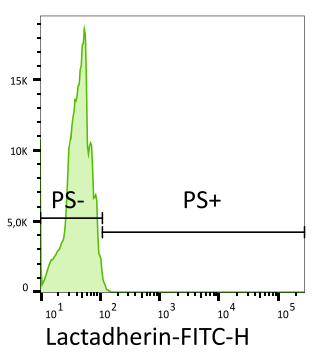

E MMV (CD14+) Labelled PFP

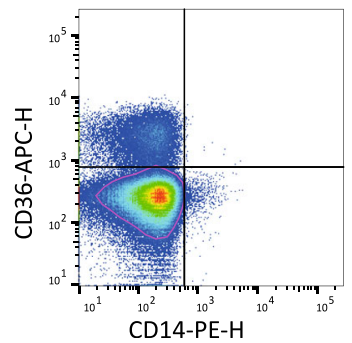

C PS Expression Labelled PFP

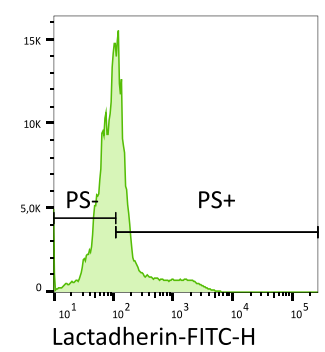

F $\operatorname{EMV}(\mathrm{CD} 62 \mathrm{E}+)$ Labelled PFP

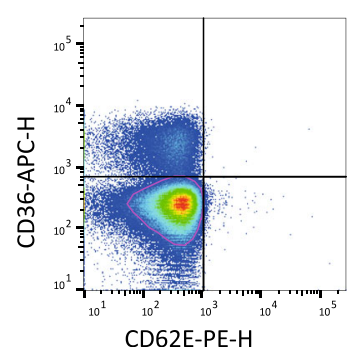

G

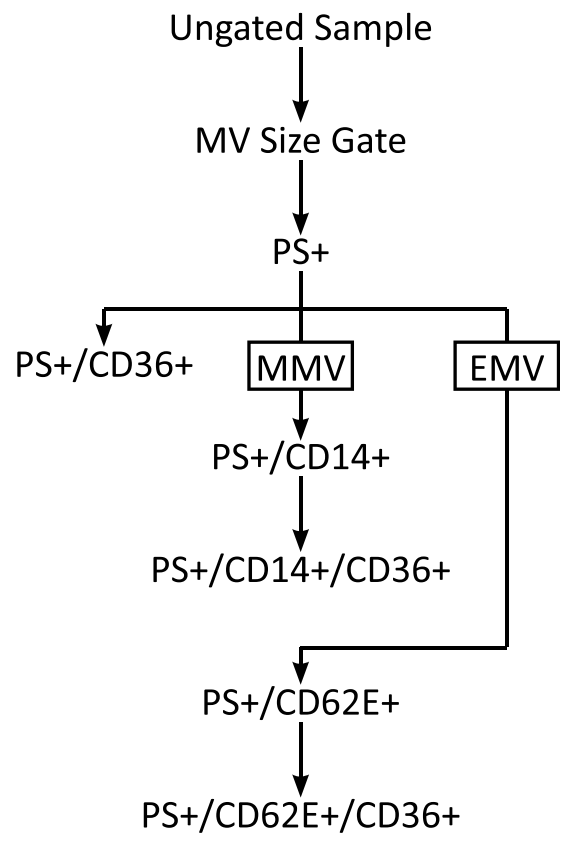

Fig. 1 Gating strategy for flow cytometric characterisation of MVs. a) A 100-1000 nm MV size gate was established based on $200 \mathrm{~nm}$ and $900 \mathrm{~nm}$ polystyrene beads (MegaMix) and transferred to all samples. b) Next, a gate was set on FITC-H at the 99th percentile of unlabelled samples. c) MVs were defined as PS+ events based on binding of lactadherin-FITC. e \& $\mathbf{f}$ ) The double negative population was defined based on density (magenta gate), and bi-variate gates were placed at the 99th percentile of the double negative population. Finally, the gates were applied to all PS+ events in the corresponding sample to define MMVs (e), EMVs (f), and the expression of CD36 on these phenotypes (e \& $\mathbf{f}$ ). $\mathbf{d})$ TruCount ${ }^{\circledR}$ beads (magenta gate) were quantified and used to calculate absolute concentrations of MVs. g) Gating hierarchy utilised for defining the MV phenotypes in the current study. PS: Phosphatidylserine; PFP: Platelet-free plasma; MMV: Monocyte microvesicles; EMV: Endothelial microvesicles

MetSy on baseline data. All $P$-values are two-sided, and statistical significance was defined as $p<0.05$.

\section{Results}

Anthropometric and biochemical characteristics

This study population has previously been described by Knøsgaard et al. [14] and data are presented in Table 1. In summary, 18 female and 2 male subjects were included into the current study, and subjects were 46.5 (range: $26 ; 63$ ) years of age at inclusion. Subjects had a median weight of $118 \mathrm{~kg}$ (IQR: 108.75; 127) and BMI of $42.1 \mathrm{~kg} \mathrm{~m}^{-2}$ (IQR: 40.4; 44.1). At the three-month post-surgical follow-up visit, subjects had incurred significant decreases in body weight (follow-up: $94 \mathrm{~kg}$ (IQR: $84.7 ; 100) ; n=20 ; p<0.0001$ ) and BMI (follow-up: $33.9 \mathrm{~kg} \mathrm{~m}^{-2}$ (IQR: 32.0; 35.0); $n=20 ; p<0.0001$ ).

\section{Effect of bariatric surgery on microvesicle phenotypes}

In the present study, plasma MV content and phenotypic origin of EVs were determined by flow cytometry. At the three-month post-surgical follow-up visit, significant decreases were observed in all of the investigated MV phenotypes with the exception of CD36+ MVs, which remained unaltered (Fig. 2 and Additional file 1: Table S2).

Specifically, concentrations of phosphatidylserine positive (PS+) MVs decreased by a median of $66.55 \%$ from baseline to follow-up ( $p=0.0017)$. This was equally accompanied by altered concentrations of MMV sub-phenotypes, where MMVs decreased by $36.11 \%$ $(p=0.0056)$ and CD36+ MMVs by $60.04 \%(p=0.0192)$. Similar results were observed for EMV sub-phenotypes, where EMV concentrations decreased by $40.10 \%$ $(p=0.0007)$ and CD36+ EMVs by $54.93 \%(p=0.04)$.

\section{Microvesicles and metabolic syndrome}

At baseline, thirteen subjects were defined as having MetSy [30], while only two subjects were defined as having MetSy at the three-month post-surgical follow-up visit. In order to investigate the impact of MetSy on MV phenotypes, subjects were initially stratified into metabolically healthy $(\mathrm{MH} ; n=7)$ and metabolically unhealthy $(\mathrm{MuH} ; n=13)$ groups based on the presence of MetSy prior to surgery (characteristics presented in Additional file 1: Table S3), and baseline concentrations of $\mathrm{MV}$ phenotypes compared between 
Table 1 Characteristics of the study population

\begin{tabular}{|c|c|c|c|c|}
\hline & Baseline & 3 monthFollow-up & $\Delta$ & $P$-value \\
\hline & $(n=20)$ & $(n=20)$ & & \\
\hline Age [Years] & $46.5 \pm 11.2$ & & & \\
\hline Sex $[M / F]$ & $2 / 18$ & & & \\
\hline Metabolic Syndrome [n (\%)] & $13(65 \%)$ & $2(10 \%)$ & & \\
\hline NASH $[n(\%)]$ & $13(65 \%)$ & $11(55 \%)$ & & \\
\hline Haemoglobin $\left[\mathrm{mmol} \mathrm{I}^{-1}\right]$ & $8.5 \pm 0.5$ & $8.4 \pm 0.6$ & $-0.1 \pm 0.4$ & 0.1405 \\
\hline Weight [kg] & $118(108.75 ; 127)$ & $94(84.7 ; 100)$ & $-23.33 \pm 4.21$ & 0.0001 \\
\hline BMI $\left[\mathrm{kg} \mathrm{m}^{-2}\right]$ & $42.1(40.4 ; 44.1)$ & $33.9(32.0 ; 35.0)$ & $-8.4 \pm 1.4$ & $<0.0001$ \\
\hline Total Fat Mass [kg] & $54.8 \pm 11.0$ & $40.2(32.9 ; 42.3)$ & $-15.6 \pm 35.6$ & $<0.0001$ \\
\hline Body Fat Percentage [\%] & $45.3 \pm 4.9$ & $39.4 \pm 6.5$ & $-5.6 \pm 2.4$ & $<0.0001$ \\
\hline Fat Mass/Fat Free Mass [AU] & $0.84 \pm 0.16$ & $0.67 \pm 0.17$ & $-0.16 \pm 0.06$ & $<0.0001$ \\
\hline Android Fat [kg] & $48.2 \pm 8.2$ & $31.9 \pm 9.6$ & $-16.8 \pm 4.7$ & $<0.0001$ \\
\hline Android Fat Percentage [\%] & $46.8 \pm 4.0$ & $39.5 \pm 6.0$ & $-7.3 \pm 3.8$ & $<0.0001$ \\
\hline Truncal Fat [kg] & $26.6 \pm 40.3$ & $17.9 \pm 49.2$ & $-8.4 \pm 2.9$ & $<0.0001$ \\
\hline Truncal Fat Percentage [\%] & $44.5 \pm 3.7$ & $37.5 \pm 6.4$ & $-6.6 \pm 3.9$ & $<0.0001$ \\
\hline Total Cholesterol $\left[\mathrm{mmol} \mathrm{I^{-1 } ]}\right.$ & $4.92 \pm 1.00$ & $4.49 \pm 0.88$ & $-0.44 \pm 0.82$ & 0.0275 \\
\hline HDL Cholesterol $\left[\mathrm{mmol} \mathrm{I}^{-1}\right]$ & $1.14 \pm 0.27$ & $1.12 \pm 0.28$ & $-0.02 \pm 0.15$ & 0.659 \\
\hline LDL Cholesterol [mmol I-1] & $2.99 \pm 0.87$ & $2.82 \pm 0.69$ & $-0.18 \pm 0.73$ & 0.2992 \\
\hline Oxidized LDL Cholesterol & $4.53 \pm 1.13$ & $4.34 \pm 0.93$ & $-0.19 \pm 0.84$ & 0.3203 \\
\hline Triglycerides $\left[\mathrm{mmol} \mathrm{I}{ }^{-1}\right]$ & $1.72 \pm 0.62$ & $1.15(1.05 ; 1.45)$ & $-0.46 \pm 0.52$ & 0.001 \\
\hline Triglyceride/HDL [AU] & $1.38(1.09 ; 1.89)$ & $1.03(0.86 ; 1.41)$ & $-0.41 \pm 0.54$ & 0.0023 \\
\hline Glycated Haemoglobin [\%] & $5.9 \pm 0.43$ & $5.6(5.5 ; 5.8)$ & $-0.14 \pm 0.40$ & 0.0792 \\
\hline Fasting Glucose [mmol I-1] & $5.45(5.18 ; 6.03)$ & $5.1(4.78 ; 5.33)$ & $-0.45(-0.8 ;-0.15)$ & 0.0157 \\
\hline Fasting C-Peptide [pmol $\mathrm{I}^{-1}$ ] & $1061(921 ; 1422)$ & $745(566 ; 935)$ & $-360(-542 ;-36.3)$ & 0.0094 \\
\hline Fasting Insulin [pmol I-1] & $131.7(97.5 ; 201.5)$ & $63.7(53.7 ; 77.8)$ & $-69.7(-105.7 ;-40.8)$ & 0.0014 \\
\hline HOMAIR & $2.55(2.05 ; 3.2)$ & $1.6(1.3 ; 2.05)$ & $-0.85(-1.43 ;-0.075)$ & 0.0149 \\
\hline HOMASE & $42.7 \pm 16.4$ & $60.4 \pm 20.7$ & $17.7 \pm 26.3$ & 0.0072 \\
\hline YKL40 $\left[\mathrm{ng} \mathrm{ml}^{-1}\right]$ & $57.5(47 ; 68.3)$ & $58(45 ; 72.8)$ & $1.1 \pm 14.8$ & 1 \\
\hline Liver Fat Percentage [\%] & $6.7(4.54 ; 9.99)$ & $2.82(2.23 ; 3.79)$ & $-2.78(-6.23 ;-1.84)$ & 0.0002 \\
\hline $\operatorname{ALT}\left[U \mathrm{I}^{-1}\right]$ & $30.9 \pm 13.6$ & $29.4 \pm 15.8$ & $-1.45 \pm 20.4$ & 0.7537 \\
\hline AST $\left[U ~^{-1}\right]$ & $28(25 ; 32.3)$ & $26(22 ; 37)$ & $0.65 \pm 12.1$ & 0.9826 \\
\hline AST/ALT [AU] & $0.97(0.88 ; 1.36)$ & $0.97(0.92 ; 1.24)$ & $0.06(-0.11 ; 0.30)$ & 0.33 \\
\hline Leukocytes $\left[\mathrm{mia}{ }^{-1}\right]$ & $8.37 \pm 2.24$ & $6.65(5.73 ; 8.08)$ & $-1.34 \pm 1.15$ & 0.0003 \\
\hline High-sensitivity CRP $\left[\mathrm{mg} \mathrm{I}^{-1}\right.$ ] & $4.67(2.78 ; 9.99)$ & $2.16(1.28 ; 5.16)$ & $-3.91 \pm 6.15$ & 0.0073 \\
\hline Soluble CD36 [AU] & $0.48 \pm 0.20$ & $0.37(0.24 ; 0.40)$ & $-0.13(-0.19 ;-0.06)$ & 0.0008 \\
\hline
\end{tabular}

Data are depicted as mean $\pm \mathrm{SD}$ or median $\left(\mathrm{Q}_{25 \%} ; \mathrm{Q}_{75 \%}\right)$

$p$-values $<0.05$ in bold text

the two groups. However, no significant differences could be observed in baseline concentrations of any of the investigated MV phenotypes between $\mathrm{MH}$ and $\mathrm{MuH}$ groups (Additional file 1: Table S3).

\section{Discussion}

Previously, in the cohort of this study, where severely obese subjects underwent bariatric surgery, we investigated how weight loss secondary to bariatric surgery affects levels of circulating sCD36 [14]. In the present study on the other hand, we investigated how different MV phenotypes specifically focussing on MMVs, EMVs and their respective phenotypes positive for the expression of CD36 are affected by weight loss following bariatric surgery. In this study, the results were two-fold. First, concentrations of total MVs, MMVs, EMVs, CD36+ MMVs and CD36+ 


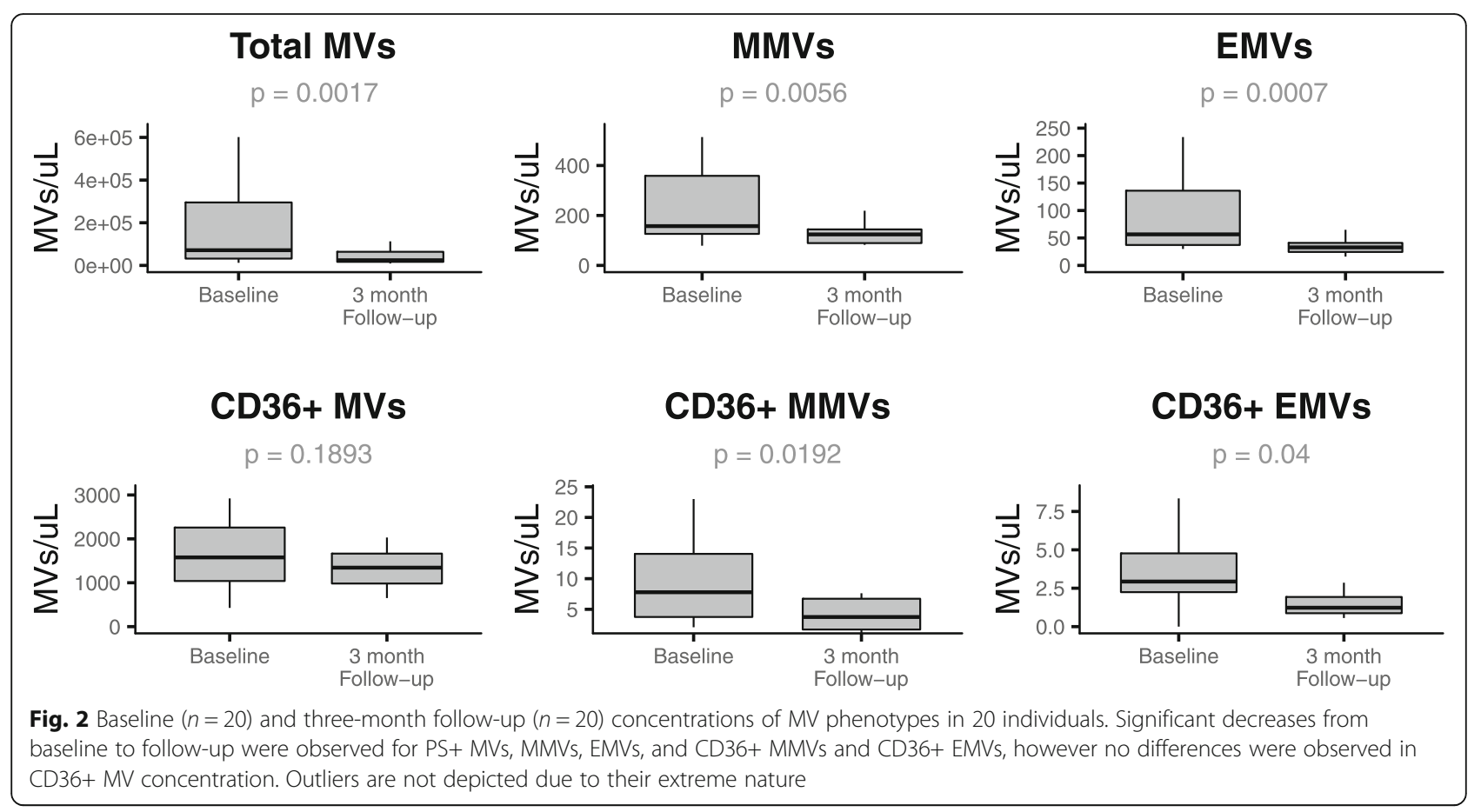

EMVs decreased significantly following bariatric surgery. Second, and somewhat conversely, there were no differences in baseline concentrations of any of the investigated MV phenotypes between $\mathrm{MH}$ and MuH subjects.

In the present study, the concentration of PS+ MVs was found to decrease significantly following bariatric surgery. In contrast to the present study, Witczak et al. described that the total number of extracellular vesicles were unaltered following bariatric surgery [31]. However, this discrepancy could be due to different pre-analytical methodology and different methods for characterising EVs, which might result in a different subset of EVs being analysed. It has previously been established that platelet MVs are the most abundant phenotype of MVs present in plasma [32], and that platelet MVs are up-regulated in obese subjects [33, 34]. Levels of platelet MVs seem to correlate with body composition [33], plasma lipids [35], and hyperglycaemia [36], all attributing to oxidative stress [37]. These parameters also seem to affect several other MV phenotypes including leukocyte MVs [38, 39], EMVs [33], and MMVs [35]. Thus, it can be inferred that the improvements observed in body composition, plasma lipid profile, and insulin sensitivity result in decreased activation of platelets, leukocytes, vascular endothelial cells, and monocytes/macrophages, which in turn could explain the significant reduction in PS+ MVs.

An interesting observation in the present study was that CD36+ MVs did not differ significantly from baseline to the three-month follow-up. This result is in contrast to a study conducted by Campello et al., in which CD36+ MVs decreased significantly from baseline to three and twelve-months follow-up visits [39]. Interestingly, although CD36+ MVs were unaltered, a significant decrease was seen in sCD36 in the present cohort. Levels of sCD36 have previously been suggested to reflect those of tissue expression [15] and further associated with unhealthy fat distribution [14], insulin resistance [15], and hepatic fat accumulation [16], all complications of obesity. It can thus be argued that sCD36 might be a more sensitive marker for cardio-metabolic complications than MV-associated CD36.

Bariatric surgery led to reductions of MMVs and CD36+ MMVs in the present study. In addition to demonstrating that bariatric surgery reduces MMVs, Cheng et al. also demonstrated that MMVs were associated with BMI and HbA1c [40]. CD36+ MMVs were further demonstrated to correlate with BMI, waist circumference, total fat mass, triglycerides, and fasting levels of C-peptide and insulin in another study [35]. A growing body of evidence is starting to recognise that macrophages play a central role in the development of insulin resistance and T2DM (reviewed in [41]). In obese individuals, steatotic cells secrete chemokines that promote migration of monocytes into tissues and their subsequent polarisation into a more pro-inflammatory phenotype $[42,43]$. Apart from this, a growing body of evidence has implicated CD36 on the surface of macrophages and its ability to interact with oxidised LDL cholesterol in the pro-inflammatory polarisation of macrophages [22]. Moreover, the expression of CD36 on the surface of macrophages is increased in obese 
individuals [44]. Thus, it is possible that the significant weight loss observed following bariatric surgery and the concurrent improvements in cardio-metabolic health leads to decreased recruitment of monocytes to steatotic tissues, increased proportion of anti-inflammatory macrophages, and overall decreased activity in macrophages. This could in turn lead to a reduction in the release of MMVs and CD36+ MMVs.

EMVs and CD36+ EMVs were found to be significantly reduced following bariatric surgery in the current study. Studies that have previously examined the impact of bariatric surgery on EMV concentrations have yielded conflicting results. In line with the present study, both Campello et al. [39] and Cheng et al. [40] reported that levels of various phenotypes of MVs including platelet MVs, EMVs and MMVs decreased significantly in obese subjects after bariatric surgery. Conversely, Stepanian et al. [34] reported no differences in platelet MVs and EMVs one year after subjects had undergone bariatric surgery, which was later supported by a study conducted by Witczak et al. [31]. However, a growing body of evidence is in support of there being a relationship between EMVs and obesity, MetSy, and T2DM [33, 45-48]. Ectopic fat deposition and hyperglycaemia have long been known to contribute to endothelial dysfunction and subsequent atherosclerosis by means of dysregulation of circulating lipids [49]. A common feature of endothelial dysfunction is the production of ROS resulting from altered intracellular metabolism [50], which in turn leads to mitochondrial fragmentation, increased endothelial permeability, and up-regulation of adhesion molecules and pro-inflammatory cytokines [51]. In turn, this promote the attachment, migration and polarisation of monocytes into underlying tissues and a subsequent inflammatory response. Some doubt has to be cast on the concentrations of EMV sub-phenotypes due to similar binding patterns of the EMV antibody and its isotype control utilised in the current assay (Additional file 2: Figure S1). It can therefore not be ruled out that the current results could have arisen from non-specific binding of the antibody and subsequent inclusion of false positive events. However, the stark differences between baseline and the three-month follow-up visit are striking nonetheless.

Somewhat controversially, baseline concentrations of MV phenotypes did not differ between subjects with and without MetSy. This result is in support of observations by Stepanian et al. [34], who also observed no differences in levels of platelet MVs and EMVs between metabolically healthy subjects and subjects with MetSy. On the other hand, Chironi et al. [38] reported that leukocyte MVs were higher in individuals with MetSy in a non-obese cohort, however EMVs did not differ between groups in their study. Conversely, Amabile et al.
[47] and Arteaga et al. [52] demonstrated that several phenotypes of EMVs were associated with the MetSy and correlated with number of components of MetSy. Although it was hypothesised that $\mathrm{MuH}$ subjects had increased levels of the investigated MV phenotypes, the authors recognise that several confounding factors might have influenced the results of the present study including BMI and body composition. To a certain extent explain, this might also differences observed between studies, as mean BMI differs greatly between studies.

A significant strength of the current study is that it employs flow cytometry to quantify and characterise MVs from subjects undergoing bariatric surgery. Although conventional flow cytometry lacks the sensitivity to measure the smallest of extracellular vesicles [53], it remains a preferred method of characterising MVs and holds great advantages over a multitude of other methods for characterising and quantifying MVs including nano-particle tacking analysis, Western blot, and ELISA. This is due to its ability to simultaneously quantify MVs and characterise their expression of multiple surface markers in a high-throughput manner, thus allowing for discrimination between large numbers of MVs with different phenotypic origins [26, 54-56]. Several limitations should, however, also be addressed in the present study. First, the authors recognise that the sample size in the present study is a limitation, which could give the study insignificant power to discover significant differences in baseline concentrations of MVs between $\mathrm{MH}$ and $\mathrm{MuH}$ subjects. In addition, this could explain the lack of correlations between changes of MV phenotypes on the one hand and body composition, plasma lipids, insulin sensitivity, and inflammatory markers on the other (data not shown). Second, the follow-up in this study is relatively short, and it is therefore impossible to make inferences about the long-term effects of bariatric surgery on levels of circulating MVs. Third, due to constraints, isotype controls were prepared for baseline samples only, thus limiting the interpretability of flow cytometric characterisation of MVs in samples from follow-up visits, as the extent of non-specific binding of antibodies to MVs cannot be examined. Nonetheless, the magnitude of decrease in MV concentrations from baseline to follow-up would arguably imply that the importance of this is minimal if not insignificant.

\section{Conclusion}

In conclusion, this study reports that concentrations of MVs and specifically those of monocyte and endothelial origin decrease significantly in the follow-up period following bariatric surgery. Moreover, the concentration of the bulk of CD36+ MVs remained unaltered, while significant decreases are seen in sCD36, CD36+ MMVs, and CD36+ EMVs. These changes likely reflect the 
significant weight loss incurred by the subjects following bariatric surgery, which in turn leads to reduction in ectopic deposition of fat, improved plasma lipid profile, decreased low-grade inflammation, and oxidative stress. At the cellular level, decreased amount of lipids in the extracellular space reduce CD36-specific uptake of lipids by endothelial cells and macrophages, which in turn reduces cell stress and CD36 expression on the cell membrane, thereby resulting in decreased shedding of MVs. However, baseline levels of MV phenotypes did not differ between subjects with MetSy and those without MetSy.

Finally, in order to further investigate the potential of MMVs, EMVs and their respective phenotypes positive for the expression of CD36, larger studies have to be conducted in which associations between their concentration and components of the metabolic syndrome and type 2 diabetes are thoroughly investigated.

\section{Additional files}

Additional file 1: Supplementary methodological information on MV characterisation by flow cytometry and antibody panels used in this study. Table S1. Antibodies and concentrations used for flow cytometric characterisation of MVs. Table S2. Baseline and three-month follow-up concentrations of MV phenotypes in study participants. Table S3. Characteristics of the metabolically healthy and metabolically unhealthy participants. (DOCX 25 kb)

Additional file 2: Figure S1. Typical scatter plots for the two different antibody panels used to characterise MV phenotypes. (EPS $1189 \mathrm{~kb}$ )

\section{Abbreviations}

ALT: alanine aminotransferase; AST: aspartate aminotransferase; BMI: Body mass index; CD: Cluster of differentiation; EMV: Endothelial-derived microvesicle; EV: Extracellular vesicle; HDL: High density lipoprotein; HOMA$\%$ S: homeostasis model assessment of sensitivity; hsCRP: Highly sensitive Creactive protein; IQR: Inter-quartile range; LDL: Low density lipoprotein; LF\%: liver fat percentage; MetSy: Metabolic syndrome; MH: Metabolically healthy; MMV: Monocyte-derived microvesicle; MuH: Metabolically unhealthy; MV: Microvesicle; NAFLD: Non-alcoholic fatty liver disease;

PS: phosphatidylserine; sCD36: Soluble CD36; T2DM: Type 2 diabetes mellitus

\section{Acknowledgements}

The authors would like to thank Dr. Charlotte Christie Petersen and the staff at the FACS Core Facility at the Institute for Biomedicine, Aarhus University for their expert assistance with setting up and performing flow cytometric analysis of all samples.

\section{Funding}

The study was supported by a grant from The Novo Nordisk Foundation (NNF13OC0007713). None of the funding bodies had any role in the design or conduct of this study nor in the preparation of this manuscript.

\section{Availability of data and materials}

The datasets used and/or analysed during the current study as well as analysis scripts are available from the corresponding author on reasonable request.

\section{Authors' contributions}

$H V$ supervised the collection of patient samples and anthropometric/body composition data at baseline and follow-up. MHC, MHN and AH were all major contributors in designing the present study. $\mathrm{MHC}$ and $\mathrm{MHN}$ designed the antibody panels and conducted flow cytometry experiments. JB analysed all data and was the major contributor in writing the manuscript. All authors approved the final version of the manuscript.

Ethics approval and consent to participate

All procedures performed in the present study involving human participants were in accordance with the 1964 Helsinki declaration and its later amendments. The Danish Data Protection Agency (id. 00908 HEK.750.86-4) and The Ethics Committee in the Capital Region of Denmark, Committee E (id. H-3-2009-100) approved the present study, and all participants gave informed written consent prior to inclusion.

\section{Consent for publication}

Not applicable.

\section{Competing interests}

Dr. Handberg reports grants from The Novo Nordisk Insulin Foundation during the conduct of the study. In addition, Dr. Handberg has a patent WO2005/116644 issued. All other authors declare that they have no competing interest pertaining to the current study.

\section{Publisher's Note}

Springer Nature remains neutral with regard to jurisdictional claims in published maps and institutional affiliations.

\section{Author details}

${ }^{1}$ Department of Clinical Biochemistry, Aalborg University Hospital, Hobrovej 18-22, DK-9000 Aalborg, Denmark. '2Department of Clinical Medicine, Faculty of Medicine, Aalborg University, Sdr. Skovvej 15, DK-9000 Aalborg, Denmark. ${ }^{3}$ Novo Nordisk Foundation Center for Basic Metabolic Research, Section of Metabolic Genetics, SUND, University of Copenhagen, Panum, Mærsk tårnet, Bygning 7, 8. Etage, DK-2200 Copenhagen N, Denmark.

Received: 14 May 2018 Accepted: 27 September 2018

Published online: 23 October 2018

\section{References}

1. Look AHEAD Research Group, Wing RR, Bolin P, Brancati FL, Bray GA, Clark $J M$, et al. Cardiovascular effects of intensive lifestyle intervention in type 2 diabetes. N. Engl. J. Med. 2013, 369:145-54 Available from: http://www.nejm. org/doi/10.1056/NEJMoa1212914

2. Li Z, Maglione M, Tu W, Mojica W, Arterburn D, Shugarman LR, et al. Metaanalysis: pharmacologic treatment of obesity. Ann. Intern. Med. 2005;142: 532-46 Available from: http://scholar.google.com/scholar?hl=en\&btnG= Search\&q=intitle:Clinical+Guidelines\#5.

3. Sjöström L. Review of the key results from the Swedish obese subjects (SOS) trial - a prospective controlled intervention study of bariatric surgery. J Intern Med. 2013;273:219-34. Available from: http://www.ncbi.nlm.nih.gov/ pubmed/23163728

4. Arterburn DE, Courcoulas AP. Bariatric surgery for obesity and metabolic conditions in adults. BMJ. 2014;349:g3961 Available from: http://www.bmj. com/cgi/doi/10.1136/bmj.g3961.

5. Thomsen SB, Rathcke CN, Jørgensen NB, Madsbad S, Vestergaard H. Effects of Roux-en-Y gastric bypass on fasting and postprandial levels of the inflammatory markers YKL-40 and MCP-1 in patients with type 2 diabetes and glucose tolerant subjects. J Obes. 2013;2013:361781. Available from: https://www.hindawi.com/journals/jobe/2013/361781/.

6. Buchwald H, Oien DM. Metabolic/bariatric surgery worldwide 2008. Obes Surg. 2009;19:1605-11 Available from: http://www.ncbi.nlm.nih.gov/ pubmed/19885707.

7. Buchwald H, Oien DM. Metabolic/bariatric surgery worldwide 2011. Obes Surg. 2013;23:427-36 Available from: http://www.ncbi.nlm.nih.gov/pubmed/ 19885707

8. Angrisani L, Santonicola A, lovino P, Vitiello A, Zundel N, Buchwald H, et al. Bariatric surgery and Endoluminal procedures: IFSO worldwide survey 2014. Obes. Surg. 2017;27:2279-89 Available from: http://www.ncbi.nlm.nih.gov/ pubmed/28405878.

9. Agouni A, Ducluzeau P-H, Benameur T, Faure S, Sladkova M, Duluc L, et al. Microparticles from patients with metabolic syndrome induce vascular hypo-reactivity via Fas/Fas-ligand pathway in mice. Reitsma PH, editor. PLoS One. 2011;6:e27809. Available from: http://www.pubmedcentral.nih. gov/articlerender.fcgi?artid=3217000\&tool=pmcentrez\&rendertype=abstract. 
10. Kurtzman N, Zhang L, French B, Jonas R, Bantly A, Rogers WT, et al. Personalized cytomic assessment of vascular health: evaluation of the vascular health profile in diabetes mellitus. Cytometry B. Clin. Cytom. 2013; 84:255-66 Available from: http://www.ncbi.nlm.nih.gov/pubmed/23740755.

11. Hjuler Nielsen M, Irvine $H$, Vedel S, Raungaard B, Beck-Nielsen H, Handberg A. Elevated atherosclerosis-related gene expression, monocyte activation and microparticle-release are related to increased lipoprotein-associated oxidative stress in familial hypercholesterolemia. Boissonnas A, editor. PLoS One . 2015;10:e0121516. Available from: http://www.pubmedcentral.nih.gov/ articlerender.fcgi?artid=4395270\&tool=pmcentrez\&rendertype=abstract.

12. Raposo G, Stoorvogel W. Extracellular vesicles: exosomes, microvesicles, and friends. J Cell Biol. 2013;200:373-83. Available from: http://www.ncbi.nlm.nih. gov/pubmed/23420871.

13. van der Pol E, Böing AN, Harrison P, Sturk A, Nieuwland R. Classification, functions, and clinical relevance of extracellular vesicles. Pharmacol Rev. 2012 64:676-705. Available from: http://www.ncbi.nlm.nih.gov/pubmed/22722893.

14. Knøsgaard L, Thomsen SB, Støckel M, Vestergaard H, Handberg A. Circulating SCD36 is associated with unhealthy fat distribution and elevated circulating triglycerides in morbidly obese individuals. Nutr. Diabetes. 2014;4 e114. Available from: http://www.pubmedcentral.nih.gov/articlerender. fcgi?artid $=4007154 \&$ tool $=$ pmcentrez\&rendertype $=$ abstract.

15. Handberg A, Levin K, Højlund K, Beck-Nielsen H. Identification of the oxidized low-density lipoprotein scavenger receptor CD36 in plasma: a novel marker of insulin resistance. Circulation. 2006;114:1169-76. Available from: http://circ.ahajournals.org/cgi/doi/10.1161/CIRCULATIONAHA.106.626135.

16. Heebøll S, Poulsen MK, Ornstrup MJ, Kjær TN, Pedersen SB, Nielsen S, et al. Circulating SCD36 levels in patients with non-alcoholic fatty liver disease and controls. Int J Obes (Lond) [Internet] Nature Publishing Group. 2017:41: 262-7. Available from: https://doi.org/10.1038/ijo.2016.223.

17. Nielsen MH, Irvine $H$, Vedel S, Raungaard B, Beck-Nielsen $H$, Handberg A The Impact of Lipoprotein-Associated Oxidative Stress on Cell-Specific Microvesicle Release in Patients with Familial Hypercholesterolemia. Oxid. Med. Cell. Longev. 2016;2016:2492858. Available from: http://www.hindawi. com/journals/omcl/2016/2492858/

18. Ghosh A, Murugesan G, Chen K, Zhang L, Wang Q, Febbraio M, et al. Platelet CD36 surface expression levels affect functional responses to oxidized LDL and are associated with inheritance of specific genetic polymorphisms. Blood. 2011;117:6355-66. Available from: http://www.ncbi. nlm.nih.gov/pubmed/21478428.

19. IOCM V, van Klinken JB, van Diepen JA, van den Berg SAA, Febbraio M, LKM S, et al. CD36 is important for adipocyte recruitment and affects lipolysis. Obesity (Silver Spring). 2013;21:2037-45. Available from: http://www.ncbi. nlm.nih.gov/pubmed/23512311.

20. Adachi H, Tsujimoto M. Endothelial scavenger receptors. Prog. Lipid Res. [Internet]. England. 2006;45:379-404 Available from: http://www.ncbi.nlm. nih.gov/pubmed/16712941.

21. Corpeleijn E, Pelsers MMAL, Soenen S, Mensink M, Bouwman FG, Kooi ME, et al. Insulin acutely upregulates protein expression of the fatty acid transporter CD36 in human skeletal muscle in vivo. J. Physiol. Pharmacol. [internet]. 2008;59:77-83. Available from: http://www.ncbi.nlm.nih.gov/ pubmed/22584574.

22. Kennedy DJ, Kashyap SR. Pathogenic role of scavenger receptor CD36 in the metabolic syndrome and diabetes. Metab. Syndr. Relat. Disord. 2011;9: 239-45. Available from: http://www.ncbi.nlm.nih.gov/pubmed/21428745.

23. Steneberg P, Sykaras AG, Backlund F, Straseviciene J, Söderström I, Edlund $H$. Hyperinsulinemia enhances hepatic expression of the fatty acid transporter Cd36 and provokes Hepatosteatosis and hepatic insulin resistance. J Biol Chem. 2015;290:19034-43. Available from: http://www.ncbi. nlm.nih.gov/pubmed/26085100.

24. Canton J, Neculai D, Grinstein S. Scavenger receptors in homeostasis and immunity. Nat. Rev. Immunol. Nature Publishing Group. 2013;13:621-34. Available from: http://www.ncbi.nlm.nih.gov/pubmed/23928573.

25. Kotronen A, Peltonen M, Hakkarainen A, Sevastianova K, Bergholm R, Johansson LM, et al. Prediction of non-alcoholic fatty liver disease and liver fat using metabolic and genetic factors. Gastroenterology. Elsevier Inc. 2009; 137:865-72. Available from: https://doi.org/10.1053/j.gastro.2009.06.005.

26. Nielsen $M H$, Beck-Nielsen $H$, Andersen MN, Handberg A. A flow cytometric method for characterization of circulating cell-derived microparticles in plasma. J. Extracell. vesicles [Internet]. 2014;3:1-12. Available from: http:// www.pubmedcentral.nih.gov/articlerender.fcgi?artid=3916676\&tool= pmcentrez\&rendertype=abstract.
27. Dragulescu AA. xIsx: Read, write, format Excel 2007 and Excel 97/2000/ XP/2003 files [Internet]. 2014. Available from: https://cran.r-project.org/ package $=x \mid s x$

28. Wickham H. ggplot2: Elegant Graphics for Data Analysis [Internet]. SpringerVerlag New York; 2009. Available from: http://ggplot2.org

29. Wickham H. Reshaping Data with the reshape Package. J. Stat. Softw. [Internet]. 2007;21. Available from: http://www.jstatsoft.org/v21/i12

30. KGMM A, Eckel RH, Grundy SM, Zimmet PZ, Cleeman JI, Donato KA, et al. Harmonizing the metabolic syndrome: a joint interim statement of the International Diabetes Federation Task Force on Epidemiology and Prevention; National Heart, Lung, and Blood Institute; American Heart Association; World Heart Federation; International. Circulation. 2009;120: 1640-5. Available from: http://www.ncbi.nlm.nih.gov/pubmed/19805654.

31. Witczak JK, Min T, Prior SL, Stephens JW, James PE, Rees A. Bariatric surgery is accompanied by changes in extracellular vesicle-associated and plasma fatty acid binding protein 4. Obes Surg. 2017:1-8. Available from: https:// link.springer.com/article/10.1007/s11695-017-2879-Z.

32. Berckmans RJ, Nieuwland R, Böing AN, Romijn FP, Hack CE, Sturk A. Cellderived microparticles circulate in healthy humans and support low grade thrombin generation. Thromb Haemost. 2001;85:639-46. Available from: http://www.ncbi.n/m.nih.gov/pubmed/11341498.

33. Helal O, Defoort C, Robert S, Marin C, Lesavre N, Lopez-Miranda J, et al. Increased levels of microparticles originating from endothelial cells, platelets and erythrocytes in subjects with metabolic syndrome: relationship with oxidative stress. Nutr. Metab. Cardiovasc. Dis. 2011;21:665-71. Available from: http://linkinghub.elsevier.com/retrieve/pii/S0939475310000128.

34. Stepanian A, Bourguignat L, Hennou S, Coupaye M, Hajage D, Salomon L, et al. Microparticle increase in severe obesity: not related to metabolic syndrome and unchanged after massive weight loss. Obesity (Silver Spring). 2013;21:2236-43. Available from: http://www.ncbi.nlm.nih.gov/pubmed/ 23512861.

35. Botha J, Velling Magnussen L, Nielsen MH, Nielsen TB, Højlund K, Andersen MS, et al. Microvesicles correlated with components of metabolic syndrome in men with type 2 diabetes mellitus and lowered testosterone levels but were unaltered by testosterone therapy. J Diabetes Res. 2017;2017:1-9. Available from: https://www.hindawi.com/journals/jdr/2017/4257875/.

36. Diamant M. Elevated Numbers of Tissue-Factor Exposing Microparticles Correlate With Components of the Metabolic Syndrome in Uncomplicated Type 2 Diabetes Mellitus. Circulation. 2002;106:2442-7 Available from: http:// circ.ahajournals.org/cgi/doi/10.1161/01.CIR.0000036596.59665.C6.

37. Furukawa S, Fujita T, Shimabukuro M, Iwaki M, Yamada Y, Nakajima Y, et al. Increased oxidative stress in obesity and its impact on metabolic syndrome. J Clin Invest. 114:1752-61. Available from: http://www.ncbi.nlm.nih.gov/ pubmed/15599400.

38. Chironi G, Simon A, Hugel B, Del Pino M, Gariepy J, Freyssinet J-M, et al. Circulating Leukocyte-Derived Microparticles Predict Subclinical Atherosclerosis Burden in Asymptomatic Subjects. Arterioscler. Thromb. Vasc. Biol. 26:2006, 2775-80. Available from: http://atvb.ahajournals.org/cgi/ doi/10.1161/01.ATV.0000249639.36915.04.

39. Campello E, Zabeo E, Radu CM, Spiezia L, Foletto M, Prevedello L, et al. Dynamics of circulating microparticles in obesity after weight loss. Intern Emerg Med. 2016;11:695-702.

40. Cheng V, Kashyap SR, Schauer PR, Kirwan JP, McCrae KR. Restoration of glycemic control in patients with type 2 diabetes mellitus after bariatric surgery is associated with reduction in microparticles. Surg. Obes. Relat. Dis. Elsevier Inc. 2013;9:207-12. Available from: https://doi.org/10.1016/j.soard.2011.09.026.

41. Castoldi A, Naffah de Souza C, Câmara NOS, Moraes-Vieira PM. The macrophage switch in obesity development. Front Immunol. 2015;6:637. Available from: http://journal.frontiersin.org/article/10.3389/fimmu.2015.00637.

42. Olefsky JM, Glass CK. Macrophages, inflammation, and insulin resistance. Annu Rev Physiol [Internet]. 2010;72:219-46. Available from: http://www. annualreviews.org/doi/10.1146/annurev-physiol-021909-135846.

43. McNelis JC, Olefsky JM. Macrophages, immunity, and metabolic disease. Immunity Elsevier Inc. 2014;41:36-48. Available from: https://doi.org/10. 1016/j.immuni.2014.05.010.

44. Kashyap SR, loachimescu AG, Gornik HL, Gopan T, Davidson MB, Makdissi A, et al. Lipid-induced insulin resistance is associated with increased monocyte expression of scavenger receptor CD36 and internalization of oxidized LDL. Obesity (Silver Spring). Nature Publishing Group. 2009;17:2142-8. Available from: http://www.pubmedcentral.nih.gov/articlerender.fcgi?artid= 2836489\&tool=pmcentrez\&rendertype=abstract. 
45. Esposito K, Ciotola M, Schisano B, Gualdiero R, Sardelli L, Misso L, et al. Endothelial microparticles correlate with endothelial dysfunction in obese women. J Clin Endocrinol Metab. 2006;91:3676-9. Available from: http:// press.endocrine.org/doi/abs/10.1210/jc.2006-0851.

46. Tramontano a F, Lyubarova R, Tsiakos J, Palaia T, Deleon JR, Ragolia L. Circulating endothelial microparticles in diabetes mellitus. Mediators Inflamm. 2010;2010:250476. Available from: http://www.ncbi.nlm.nih.gov/ pubmed/20634911.

47. Amabile N, Cheng S, Renard JM, Larson MG, Ghorbani A, McCabe E, et al. Association of circulating endothelial microparticles with cardiometabolic risk factors in the Framingham Heart Study. Eur. Heart J. 2014;35:2972-9. Available from: http://www.ncbi.nlm.nih.gov/pubmed/24742886.

48. Agouni A, Lagrue-Lak-Hal AH, Ducluzeau PH, Mostefai HA, Draunet-Busson C, Leftheriotis G, et al. Endothelial dysfunction caused by circulating microparticles from patients with metabolic syndrome. Am. J Pathol. 2008; 173:1210-9. Available from: http://linkinghub.elsevier.com/retrieve/pii/ S000294401061509X.

49. Tchernof A, Despres J-P. Pathophysiology of human visceral obesity: an update. Physiol Rev. 2013;93:359-404. Available from: http://physrev physiology.org/cgi/doi/10.1152/physrev.00033.2011.

50. Brownlee $M$. The pathobiology of diabetic complications: a unifying mechanism. Diabetes. 2005:54:1615-25. Available from: http://www.ncbi. nlm.nih.gov/pubmed/15919781.

51. Jiang Y, Jiang L-LI, Maimaitirexiati X-MZY, Zhang Y, Wu L. Irbesartan attenuates TNF-a-induced ICAM-1, VCAM-1, and E-selectin expression through suppression of NF-KB pathway in HUVECs. Eur. Rev. med. Pharmacol. Sci. 2015;19:3295-302. Available from: http://www.ncbi.nIm.nih. gov/pubmed/26400537.

52. Arteaga RB, Chirinos JA, Soriano AO, Jy W, Horstman L, Jimenez JJ, et al. Endothelial microparticles and platelet and leukocyte activation in patients with the metabolic syndrome. Am. J Cardiol. 2006;98:70-4. Available from: http://www.ncbi.nlm.nih.gov/pubmed/16784924.

53. Lacroix R, Robert S, Poncelet P, Dignat-George F. Overcoming limitations of microparticle measurement by flow cytometry. Semin Thromb Hemost. 2010;36:807-18. Available from: http://www.ncbi.nlm.nih.gov/pubmed/ ?term $=21049381$.

54. Arraud N, Gounou C, Linares R, Brisson AR. A simple flow cytometry method improves the detection of phosphatidylserine-exposing extracellular vesicles. J Thromb Haemost. 2015;13:237-47. Available from: http://doi.wiley. com/10.1111/jth.12767.

55. Arraud N, Gounou C, Turpin D, Brisson AR. Fluorescence triggering: A general strategy for enumerating and phenotyping extracellular vesicles by flow cytometry. Cytometry. 2016;89:184-95. Available from: http://www.ncbi. nlm.nih.gov/pubmed/25857288

56. Pospichalova V, Svoboda J, Dave Z, Kotrbova A, Kaiser K, Klemova D, et al. Simplified protocol for flow cytometry analysis of fluorescently labeled exosomes and microvesicles using dedicated flow cytometer. J. Extracell. vesicles 2015:4:25530. Available from: http://www.pubmedcentral.nih.gov/ articlerender.fcgi?artid $=4382613 \&$ tool $=$ pmcentrez\&rendertype $=$ abstract.

Ready to submit your research? Choose BMC and benefit from:

- fast, convenient online submission

- thorough peer review by experienced researchers in your field

- rapid publication on acceptance

- support for research data, including large and complex data types

- gold Open Access which fosters wider collaboration and increased citations

- maximum visibility for your research: over $100 \mathrm{M}$ website views per year

At $\mathrm{BMC}$, research is always in progress.

Learn more biomedcentral.com/submissions 